\title{
Факторная модель переднеазиатской депрессии на изобарическом уровне 925 гПа
}

\author{
Ю. В. Шипко ${ }^{1}$ В. С. Балакин ${ }^{1}$, Е. В. Шувакин ${ }^{2 \bowtie}$ \\ ${ }^{1} Ц$ центральный научно-исследовательский институт Военно-воздушных сил (Минобороны России), \\ Российская Федерачия (141103, г. Щелково, Московская обл., Аэродромная ул., 2) \\ ${ }^{2}$ ВУНЦ ВВС «Военно-воздушная академия имени проф. Н. Е. Жуковского и Ю. А. Гагарина», \\ Российская Федераиия (394064, г. Воронеж, Старых Большевиков ул., 54A) \\ Поступила в редакиию 24.09.2019 \\ Принята к публикации 26.02.2020
}

\begin{abstract}
Аннотация: В статье рассматривается сезонный центр действия атмосферы - переднеазиатская депрессия - преобладающий тип атмосферной циркуляции нижней тропосферы в летний период над территорией Ближнего и Среднего Востока. Характеристики депрессии обусловливают погодно-климатические условия региона, которые могут ограничивать действия различных потребителей метеорологической информации. Цель работы - выявление особенностей внутренней структуры депрессии. Maтериальl и методbl. Для достижения поставленной цели проведен статистический анализ многомерных данных реанализа параметров атмосферы (периода 1988-2017 гг.). Использованы методы факторного анализа, кластеризации, корреляционного анализа.

На первом этапе построена модель переднеазиатской депрессии на изобарическом уровне 925 гПа, включающая 10 предикторов; после сжатия информации сделан переход к факторной модели. Резульmaты. Выявлены кластеры (типы) объектов циркуляции, представлена визуализация кластеров на факторной плоскости, дана оценка информативности факторов для приземной температуры воздуха в летние месяцы.

Модельные характеристики структурных объектов общей циркуляции атмосферы могут быть использованы в качестве предикторов для диагноза и прогноза требуемой метеорологической информации. Полученные в работе новые сведения расширяют представление о внутренней структуре летней циклонической циркуляции над территорией Ближнего и Среднего Востока.
\end{abstract}

Ключевые слова: переднеазиатская депрессия, геопотенциальная высота, реанализ, изобарический уровень, фактор, кластер, коэффициент корреляции.

\section{Factorial Model of South Asia Low at Isobaric Level 925 hPa}

\section{Yu. V. Shipko1, V.S. Balakin ${ }^{1}$, Y. V. Shuvakin ${ }^{2 凶}$}

\begin{abstract}
The paper examines seasonal atmospheric center of action - the South Asia Low, the predominant type of atmospheric circulation of the lower troposphere during the summer over the territory of the Near and Middle East. The characteristics of depression determine the weather and climatic conditions of the region, which may limit the actions of various consumers of meteorological information. The purpose of the work was to identify the features of the internal structure of depression. To achieve it, a statistical analysis of multidimensional data (period 1988-2017) of reanalysis of atmospheric parameters was carried out. The methods of factor analysis, clustering and correlation are used.

In the first stage, model of the South Asia Low at $925 \mathrm{hPa}$ level, including 10 predictors, was constructed; after information compression, transition to the factor model was made. As a result, clusters (types) of circulation objects were identified; the visualization of clusters on the factorial plane was presented; information value of common factors for surface air temperature in summer months was estimated.
\end{abstract}

() Шипко Ю.В., Балакин В.С., Шувакин Е.В., 2020

$\triangle$ E-mail: argentina 85@mail.ru

(i) Контент доступен под лицензией Creative Commons Attribution 4.0 License.

The content is available under Creative Commons Attribution 4.0 License. 
Model characteristics of structural objects of the general atmospheric circulation can be used as predictors for the diagnosis and forecast of the required meteorological information. The new information obtained in the work broadens the idea of the internal structure of the summer cyclonic circulation over the territory of the Near and Middle East.

Key words: South Asia Low, geopotential height, reanalysis, isobaric level, factor, cluster, correlation coefficient.

\section{ВВЕДЕНИЕ}

В основе метеорологического обеспечения различных потребителей лежит знание закономерностей процессов циркуляции атмосферы, обусловливающих характер погоды и ее изменений. В статье рассматривается основное звено атмосферной циркуляции нижней тропосферы над территорией Ближнего и Среднего Востока в теплый период года - сезонный центр действия атмосферы - переднеазиатская депрессия (ПАД) [2]. ПАД - термическая депрессия, имеет устойчивый сезонный характер, начинает формироваться в апреле, достигает максимального развития в июле, августе, когда значительно возрастает солнечная радиация, что приводит к сильному нагреванию подстилающей поверхности.

С образованием ПАД связано возникновение этезий - преобладающих северных ветров в восточной части Средиземного моря, дующих с большим постоянством с апреля по октябрь. Кроме того, термические вихри летнего сезона создают большую запыленность воздуха, снижающую видимость, временами до 100 м и менее. После пыльной бури продолжительное время наблюдается сухая мгла, которая нередко распространяется до высоты 6-10 км. Повторяемость сухих туманов в июле составляет $15-25 \%$.

Отмеченные метеорологические условия ограничивают или исключают реализацию метеозависимых решений различных потребителей. Таким образом, актуальность разработки модели ПАД (с выявлением особенностей атмосферной циркуляции нижней тропосферы) - для оценки ее влияния на погодно-климатические характеристики и совершенствования прогностических схем, не вызывает сомнений.

\section{МАТЕРИАЛЫ И МЕТОДЫ ИССЛЕДОВАНИЯ}

Использовался архивный материал резервного объективного анализа (реанализа) параметров атмосферы NCEP/DOE AMIP-II [7] за летние месяцы (июнь, июль, август) 30-летнего периода 1988-2017 гг. Рассматривались файлы данных срочных значений $(00,06,12,18$ ч Всемирного скоординированного времени) геопотенциальной высоты изобарического уровня 925 гПа и приземной температуры воздуха уровня 1000 гПа. Предвари- тельно проведен расчет среднесуточных значений в узлах регулярной сетки с шагом $2,5^{\circ}$ по широте и долготе. Применена программа автоматизированной обработки файлов реанализа NCEP/DOE.

\section{Базовая модель переднеазиатской депрессии}

Депрессия прослеживается до высоты 3-4 км. Для оценки ее влияния на погодно-климатические характеристики построена программно-реализованная модель на изобарическом уровне 925 гПа. Рассматривается поверхность $H_{925}$, ограниченная по широте $\varphi=10-40^{\circ}$ и долготе $\lambda=30-80^{\circ}$, представленная дискретными значениями $H_{i j}$ в узлах регулярной сетки с шагом $2,5^{\circ}$.

Применен подход, определяющий циклонический объект как объем «чаши» (с жидкостью), образованной топографией изобарической поверхности с краевой изогипсой $H_{0}$ и горизонтальной плоскостью, проходящей через эту изогипсу. Краевая изогипса определяется по алгоритму [1]: 1) нахождение минимального значения геопотенциальной высоты $H_{\text {min }}$ в рассматриваемой области; 2) определение значения $H_{0}=H_{\min }+4$, которое принимается за краевую изогипсу (учитывается, что изогипсы принято проводить через 4 дам).

В базовой модели ПАД объект циркуляции описывается параметрами: $H_{\text {min }}$; координаты центра масс «чаши» $\varphi_{c}, \lambda_{c}$; площадь $S$, ограниченная краевой изогипсой; условная «масса» $M$ и плотность $Z=M / S$ рассматриваемого объема; характеристики эллипса рассеяния условных масс объектов циркуляции $\sigma_{u}, \sigma_{v}, k$; угол положения оси ложбины $\beta$ [2].

Программная реализация модели ПАД позволила провести вычислительный эксперимент, в результате которого получена выборка (объема $n=2760)$ циклонических образований. Результаты статистической обработки многомерной выборки представлены в таблице 1.

\section{Факторная модель переднеазиатской депрессии}

С целью сжатия информации использован подход факторного анализа, когда параметры модели ПАД $(j=1,2, \ldots, m)$ представляются в виде линейной комбинации небольшого числа $k<m$ скрытых общих факторов: 
Статистические оценки параметров модели (уровень 925 гПа, июнь-август, 1988-2017 гг.)

[Table 1. Statistical estimates of model parameters (925 hPa level, June-august, 1988-2017)]

\begin{tabular}{|l|c|c|c|c|c|}
\hline $\begin{array}{c}\text { Параметр объекта } \\
\text { [Parameter of object] }\end{array}$ & $\begin{array}{c}\text { Средняя } \\
\text { [Mean] }\end{array}$ & $\begin{array}{c}\text { Медиана } \\
\text { [Median] }\end{array}$ & $\begin{array}{c}\text { Максимум } \\
\text { [Maximum] }\end{array}$ & $\begin{array}{c}\text { Минимум } \\
\text { [Minimum] }\end{array}$ & $\begin{array}{c}\text { Среднее квадр. } \\
\text { отклонение } \\
\text { [Standard deviation] }\end{array}$ \\
\hline$H_{\min }$, дам & 66,59 & 66,53 & 72,93 & 60,00 & 2,22 \\
\hline$S \cdot 10^{-6}$, км $^{2}$ & 3,8630 & 3,5300 & 12,9911 & 0,54499 & 2,0480 \\
\hline$M \cdot 10^{-6}$, км $^{2}$ дам & 5,5588 & 4,7371 & 25,0693 & 0,0110 & 3,3698 \\
\hline$Z$, дам & 1,42 & 1,42 & 2,38 & 0,70 & 0,24 \\
\hline$\sigma_{u}$, км & 779,7 & 778,5 & 1611,3 & 135,8 & 256,2 \\
\hline$\sigma_{v}$, км & 156,3 & 144,8 & 453,2 & 61,2 & 62,2 \\
\hline$k$ & 0,21 & 0,19 & 0,90 & 0,07 & 0,07 \\
\hline$\lambda_{c}$, град. в. д. & 64,07 & 64,34 & 79,07 & 41,86 & 5,45 \\
\hline$\varphi_{c}$, град. с. ш. & 28,62 & 28,69 & 34,87 & 7,19 & 1,53 \\
\hline$\beta$, град. & 132,21 & 132,83 & 146,97 & 84,81 & 6,17 \\
\hline
\end{tabular}

Факторные нагрузки параметров модели

Таблица 2

[Table 2. Factor loads of model parameters]

\begin{tabular}{|l|c|c|c|}
\hline \multirow{2}{*}{$\begin{array}{c}\text { Параметр объекта } \\
\text { [Parameter of object] }\end{array}$} & \multicolumn{3}{|c|}{ Фактор модели [Factor of model] } \\
\cline { 2 - 4 } & $f_{1}$ & $f_{2}$ & $f_{3}$ \\
\hline$H_{\min }$, дам & 0,7263 & $-0,1039$ & 0,1540 \\
\hline$S \cdot 10^{-6}$, км $^{2}$ & 0,9588 & 0,1052 & $-0,1709$ \\
\hline$M \cdot 10^{-6}$, км $^{2}$ дам & 0,8775 & 0,1144 & $-0,4125$ \\
\hline$Z$, дам & $-0,0073$ & 0,0885 & $-0,9078$ \\
\hline$\sigma_{u}$, км & 0,9053 & $-0,1263$ & 0,1876 \\
\hline$\sigma_{v}$, км & 0,8115 & 0,5047 & $-0,1158$ \\
\hline$k$ & $-0,0498$ & 0,8271 & $-0,3209$ \\
\hline$\lambda_{c}$, град. в. д. & $-0,6921$ & $-0,5059$ & 0,0133 \\
\hline$\varphi_{c}$, град. с. ш. & $-0,1100$ & 0,6478 & 0,3625 \\
\hline$\beta$, град. & $-0,2619$ & $-0,8371$ & 0,1064 \\
\hline
\end{tabular}

$$
z_{j}=a_{j 1} \cdot f_{1}+a_{j 2} \cdot f_{2}+\ldots+a_{j k} \cdot f_{k}+e_{j},
$$

где $z_{j}$ - признаки объекта (центрированные и нормализованные параметры); $a_{j k}-$ коэффициенты факторные нагрузки; $f_{i}(i=1,2, \ldots, k)$ - общие факторы; $e_{j}$ - специфичный фактор (остаток).

Сделан переход от пространства десяти признаков объектов депрессии к 3-мерному пространству ортогональных факторов. Выделенные общие факторы объясняют $\approx 80 \%$ общей дисперсии признаков.
В таблице 2 представлены полученные факторные нагрузки, где выделены значения близкие или превышающие по модулю 0,7 .

На основании данных таблицы 2 (значений факторных нагрузок) факторам модели предлагается следующая интерпретация:

$-f_{1}$ - фактор мощности ПАД (47\% общей дисперсии признаков);

- $f_{2}$ - фактор вытянутости ПАД с учетом ложбин (21\% дисперсии);

$-f_{3}-$ фактор интенсивности депрессии (12\%). 


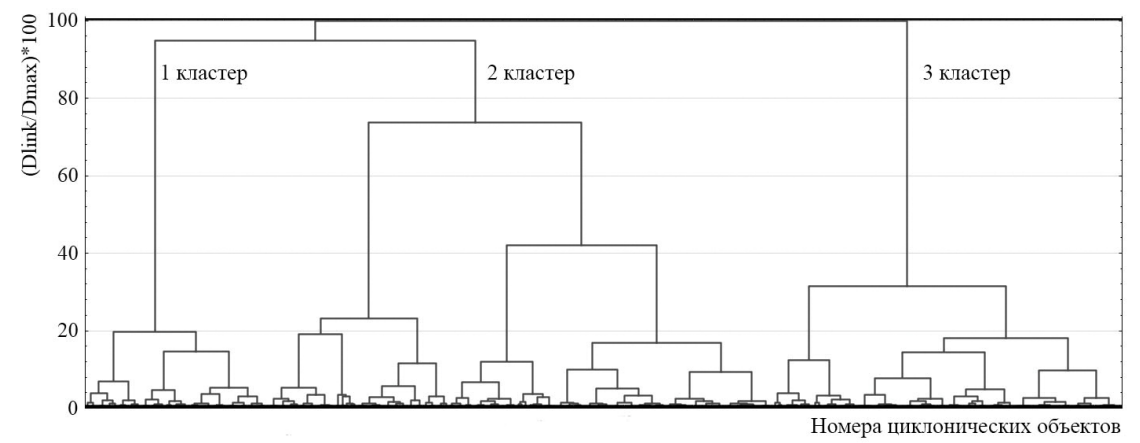

Puc. 1. Дерево иерархической кластеризации в пространстве объектов ПАД [Fig. 1. Tree of hierarchical clustering in space of objects SAL]

Полученные результаты факторной модели ПАД могут служить основой при разработке регрессионных моделей параметров атмосферы (в дальнейшем исследовании влияния характеристик депрессии на метеорологические величины), в которых предикторы являются некоррелированными, что повышает качество статистических и прогностических характеристик моделей.

\section{Оценка внутренней структуры переднеазиатской депрессии}

С целью определения однородных по структуре групп циркуляционных образований ПАД проведена процедура иерархической кластеризации объектов многомерного пространства (по выборке суточных значений характеристик). При этом не требуется априорной информации о распределении генеральной совокупности объектов. Мерой близости циклонических объектов депрессии выбрано евклидово расстояние:

$$
D=\sqrt{\left(x_{1 i}-x_{1 j}\right)^{2}+\left(x_{2 i}-x_{2 j}\right)^{2}+\ldots+\left(x_{m i}-x_{m j}\right)^{2}},
$$

где $x_{p l}$ - компоненты вектора наблюдений ( $p-$ индекс признака, $p=1,2, \ldots, m ; l-$ индекс объекта, $l=1,2, \ldots, n ; m$ - число признаков, $n$ - объем выборки).

На рисунке 1 отображено дерево кластеризации объектов ПАД, где отчетливо выделяются три ветви сгустков.

Визуализация полученного кластеризацией разделения общей совокупности циркуляционных объектов ПАД представлена на плоскости выделенных общих факторов $f_{1}, f_{2}$ (рис. 2 , где обозначены объекты левой и правой ветвей «дерева»: кластеров 1 и 3). На рисунке 2 не показан кластер 2 , чтобы не затенять картину разделения кластеров 1, 3 (тип 1 и тип 3 ).

Как видно на рисунке 2 , по фактору $f_{1}$ (мощности депрессии) хорошо дифференцируются объекты кластеров 1 и 3 (тип 1 - более «мощные» объекты).

На рисунке 3 дано распределение всех объектов циркуляции ПАД (трех кластеров) на плоскости факторов $f_{1}, f_{3}$.

На рисунке 4 представлены центры циркуляции $\left(\varphi_{c}, \lambda_{c}\right)$ циклонических объектов ПАД для выделенных кластеров 1, 3 на координатной плоскости.

Как следует из рисунков 2 и 4, циркуляционные образования ПАД кластера 1 (более мощные по фактору $f_{1}$ ) имеют центры циркуляции в западной части рассматриваемого макрорегиона, а кластера 3 (с меньшей мощностью) - в восточной части.

Средние значения общих факторов по рассматриваемым типам (кластерам) циркуляции следующие:

1 тип ПАД (кластер 1):

$$
\overline{f_{1}}=1.384 ; \overline{f_{2}}=-0.119 ; \overline{f_{3}}=-0.710 ;
$$

2 тип ПАД (кластер 2):

$$
\overline{f_{1}}=-0.020 ; \overline{f_{2}}=0.279 ; \overline{f_{3}}=0.659 ;
$$

3 тип ПАД (кластер 3):

$$
\overline{f_{1}}=-0.694 ; \overline{f_{2}}=-0.342 ; \overline{f_{3}}=-0.584 \text {. }
$$

Установлена следующая повторяемость типов $(1,2,3)$ ПАД по месяцам: июнь - 9,6; 42,3; 48,1 \%; июль - 11,7; 53,2; 35,1\%; август - 31,2; 50,5; $18,3 \%$.

По этим данным можно сделать вывод, что от июня к августу растет повторяемость циркуляционных объектов ПАД 1 типа, что соответствует, в основном, положительным значениям фактора $f_{1}$ (рис. 2), и снижается повторяемость 3 типа (на рис. 2 - отрицательные значения $f_{1}$ ), что согласуется с положением развития термической депрессии в течение летнего сезона.

\section{Оценка информативности факторов модели}

Проведена оценка информативности факторов модели ПАД уровня 925 гПа с целью возможности их использования в прогностических схемах 


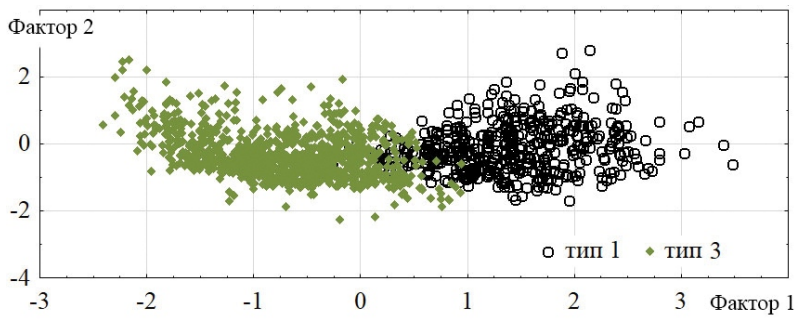

Puc. 2. Отображение кластеров на плоскости факторов $f_{1}, f_{2}$ (без учета кластера 2)

[Fig. 2. Representing clusters on the factor plane $f_{1}, f_{2}$ (without cluster 2)]

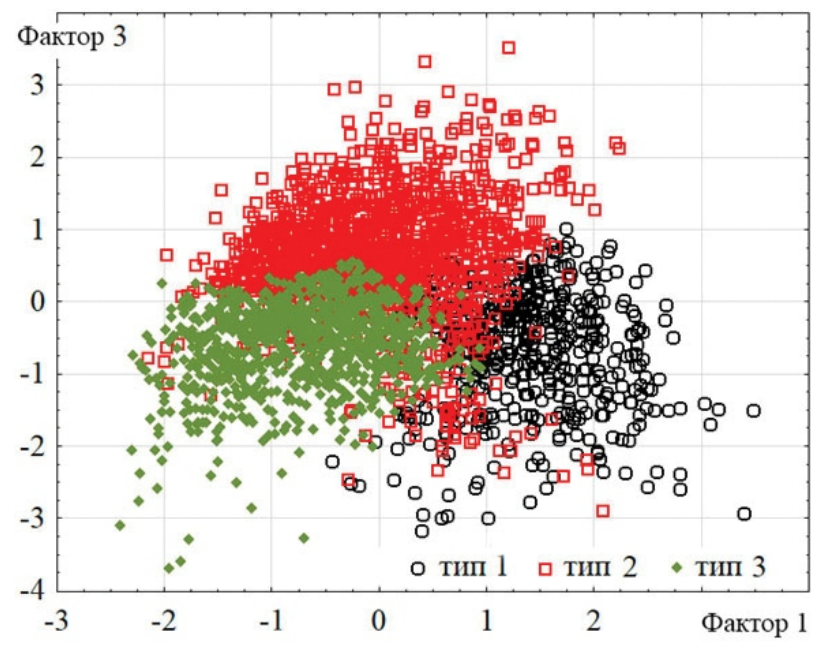

Puc. 3. Отображение выделенных кластеров на плоскости факторов $f_{1}, f_{3}$

[Fig. 3. Representing clusters on the factor plane $f_{1}, f_{3}$ ]

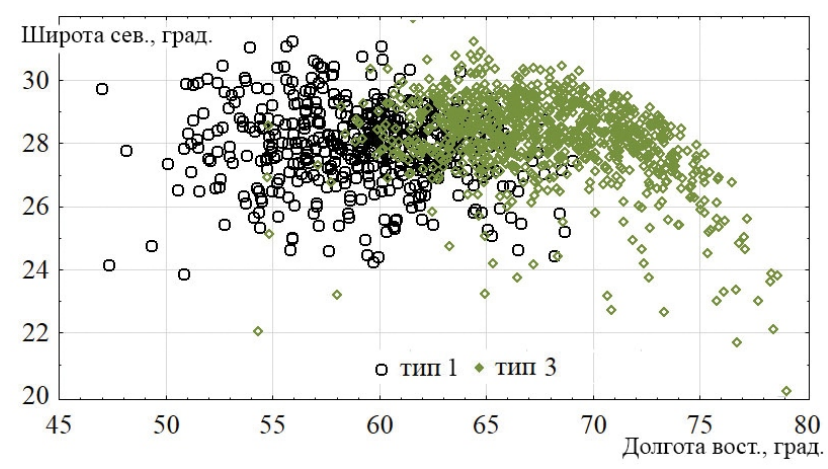

Puc. 4. Отображение центров циркуляции $\left(\varphi_{c}, \lambda_{c}\right)$ кластеров 1, 3

[Fig. 4. Representing the centers of circulation $\left(\varphi_{c}, \lambda_{c}\right)$ clusters 1,3 ]

приземной температуры воздуха. В качестве показателя информативности использовался коэффициент корреляции между факторами $f_{k}(k=1,2,3)$ и температурой воздуха $t_{i j}$ в узлах $\left(\varphi_{i}, \lambda_{j}\right)$ регулярной сетки уровня 1000 гПа:

$$
r=\frac{1}{n} \sum_{l=1}^{n}\left(\frac{f_{k l}-\overline{f_{k}}}{\sigma_{f k}}\right)\left(\frac{t_{i j l}-\overline{t_{i j}}}{\sigma_{t i j}}\right),
$$

где $f_{k l}, t_{i j l}$ - значения $l(l=1,2, \ldots, n)$ в выборке фактора $f_{k}$ и температуры $t_{i j} ; \overline{f_{k}}, \overline{t_{i j}}, \sigma_{f k}, \sigma_{t i j}-$ средние и средние квадратические отклонения параметров.

Чтобы оценить значимость оценки коэффициента корреляции и, соответственно, реальность измеряемой связи между температурой воздуха и факторами ПАД, проверяется гипотеза о равенстве коэффициента корреляции нулю. Для принятия нулевой гипотезы: $r=0$, используется условие:

$$
\left|r^{*}\right| \sqrt{n-2} / \sqrt{1-r^{* 2}}<t_{\kappa p}(\alpha, n),
$$

где $t_{\kappa p}(\alpha, n)$ - критическая точка распределения Стьюдента с $v=n-2$ степенями свободы уровня значимости $\alpha$.

Определено, что нулевая гипотеза отвергается при $|r *|>0,07$ (при уровне значимости $\alpha=0,025$ и объемах выборки $n>900$ (объем $n \geq 900$ - для одного летнего месяца 30-летнего периода). Таким образом, условно можно считать информативными характеристики ПАД, для которых выборочный коэффициент корреляции с температурой воздуха $|r *|>0,07$.

Выполнен расчет корреляционной зависимости факторов модели и приземной температуры воздуха (уровня 1000 гПа) для синхронной связи. На рисунках 5, 6 отражены поля распределения коэффициента корреляции в виде $|r *|$ на карте Ближнего Востока для летнего сезона.

Как следует из анализа полей информативности (рис. 5, 6), влияние на приземную температуру воздуха каждого фактора различается и по степени, и по районам Ближнего Востока, и по площади этих районов. Поэтому к использованию того или иного фактора в практике моделирования климатической системы или построения прогностических зависимостей следует подходить дифференцированно.

\section{ЗАКЛЮЧЕНИЕ}

Циркуляция атмосферы - главный фактор, оказывающий влияние на формирование и пространственное распределение всех метеорологических параметров. Поэтому модельные характеристики структурных объектов общей циркуляции атмосферы можно использовать в качестве предикторов для диагноза и прогноза требуемой метеорологической информации. 


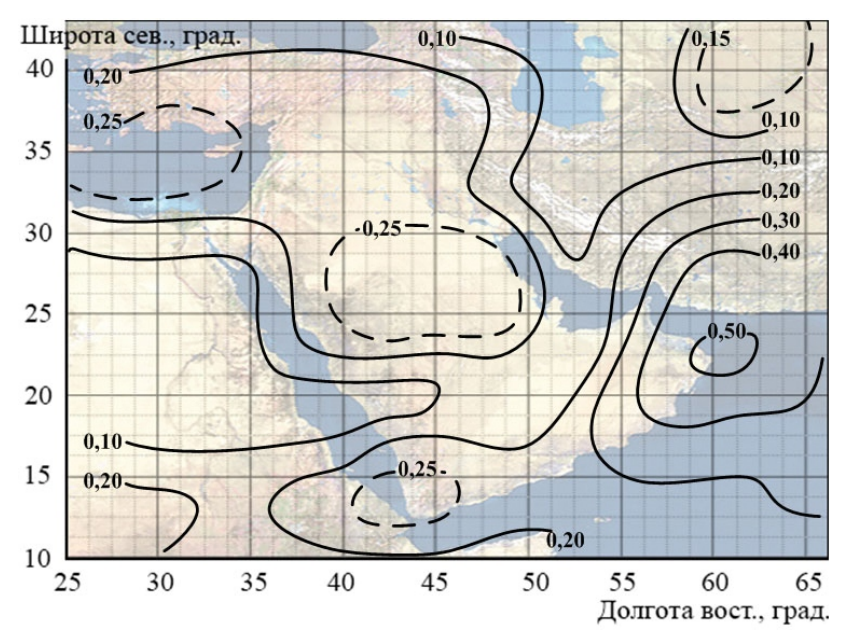

Puc. 5. Поле информативности фактора $f_{1}$ (июнь-август, 1988-2017 гг.)

[Fig. 5. Factor $f_{1}$ information field (June-August, 1988-2017)]

Полученные в статье новые сведения расширяют представление о внутренней структуре циркуляции над территорией Среднего и Ближнего Востока в летний период. Проведенное исследование является предварительным этапом многомерного статистического анализа циклонических объектов ПАД.

Достоверность полученных результатов обоснована привлечением большого объема исходных данных, применением классических методов многомерной статистической обработки материала, использованием программной реализации расчетных алгоритмов.

\section{СПИСОК ЛИТЕРАТУРЫ}

1. Шипко Ю. В., Бучнев Д. В., Облогин М. А., Балабан О.Р. Модель азиатской депрессии летнего периода на изобарическом уровне 1000 гПа // Воздушно-космические силы. Теория и практика, 2018, № 8. Доступно: http://www.vva.mil.ru/izdaniay/vks-teoriya-i-praktika (дата обращения: 22.05.2018).

2. Шипко Ю. В., Шувакин Е. В., Шуваев М. А. Модель циркумполярного вихря северного полушария в терминах механики // Материалы XVII Международ-

\section{КОНФЛИКТ ИНТЕРЕСОВ}

Авторы декларируют отсутствие явных и потенциальных конфликтов интересов, связанных с публикацией настоящей статьи.

Шипко Юрий Владимирович

кандидат технических наук, доцент, старший научный сотрудник, Центральный научно-исследовательский институт Военно-воздушных сил (Минобороны Рос-

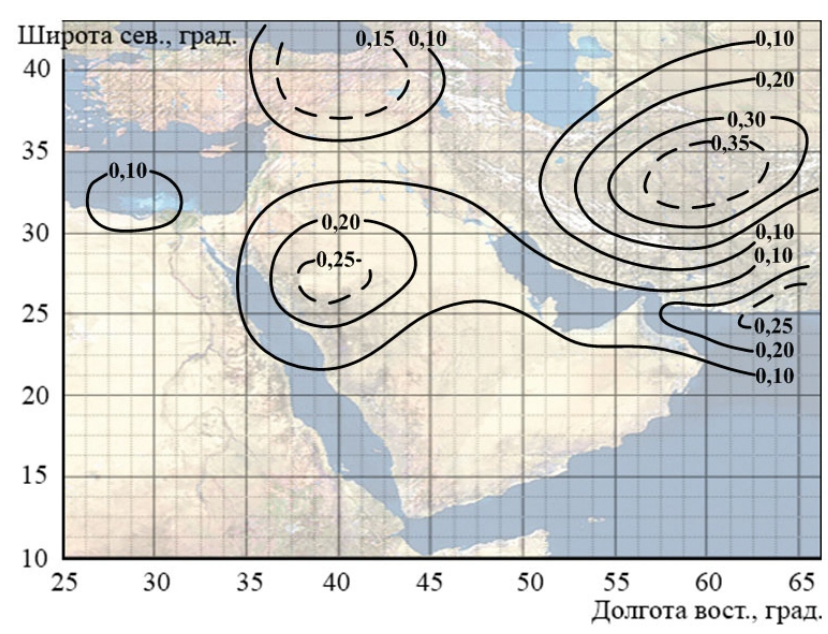

Рис. 6. Поле информативности фактора $f_{2}$ (июнь-август, 1988-2017 гг.)

[Fig. 6. Factor $f_{2}$ information field (June-August, 1988-2017)]

ной научно-методической конференции «Информатика: проблемы, методология, технологии». Воронеж, Научно-исследовательские публикации, 2017, т. 4, с. 91-96.

3. NCEP/DOE AMIP-II Reanalysis. Доступно: http:// www.esrl.noaa.gov/pcd/data/gridded/data.ncep.reanalysis2.html (дата обращения: 22.05.2018).

\section{REFERENCES}

1. Shipko Yu. V., Buchnev D. V., Oblogin M. A., Balaban O.R. [Summer period asian depression model at $1000 \mathrm{hPa}$ level]. Vozdushno-kosmicheskie sily. Teoriya $i$ praktika, 2018, no. 8. (In Russ.) Available at: http:// www.vva.mil.ru/izdaniay/vks-teoriya-i-praktika (accessed: 22.05.2018).

2. Shipko Yu. V., Shuvakin E. V. Shuvaev M. A. [Northern Hemisphere circumpolar vortex model in terms of mechanics]. Materialy XVII Mezhdunarodnoy nauchno-metodicheskoy konferentsii "Informatika: problemy, metodologiya, tekhnologii” [Proc. XVII Int. sci.-method. conf. "Informatics: problems, methodology, technologies"]. Voronezh, Nauchno-issledovatel'skie publikatsii, 2017, v. 4, pp. 91-96. (In Russ.)

3. NCEP/DOE AMIP-II Reanalysis. Available at: http:// www.esrl.noaa.gov/pcd/data/gridded/data.ncep.reanalysis2.html (accessed: 22.05.2018).

\section{CONFLICT OF INTEREST}

The authors declare no information of obvious and potential conflicts of interest related to the publication of this article.

Yuriy V. Shipko

Cand. Sci. (Tech.), Assoc. Prof., Senior researcher, Central Scientific Research Institute of Air Force (RF Ministry of Defense), Shelkovo, Moscow reg., Russian Federation, 
сии), г. Щелково, Московская обл., Российская Федерация, ORCID: https://orcid.org/0000-0002-3677-6594, E-mail: yshipko@mail.ru

Балакин Владимир Станиславович старший научный сотрудник, Центральный научно-исследовательский институт Военно-воздушных сил (Минобороны России), г. Щелково, Московская обл., Российская Федерация, ORCID: https://orcid.org/0000-0002-7332-2946, E-mail: balakin69vs@gmail.com

Шувакин Евгений Витальевич кандидат технических наук, преподаватель, Военный учебно-научный центр ВВС «Военно-воздушная академия им. профессора Н. Е. Жуковского и Ю. А. Гагарина», г. Воронеж, Российская Федерация, ORCID: https://orcid.org/0000-0002-1719-9592, E-mail: argentina 85@mail.ru
ORCID: https://orcid.org/0000-0002-3677-6594, E-mail: yshipko@mail.ru

\section{Vladimir S. Balakin}

Senior researcher, Central Scientific Research Institute of Air Force (RF Ministry of Defense), Shelkovo, Moscow reg., Russian Federation, ORCID:

https://orcid.org/0000-0002-7332-2946, E-mail: balakin69vs@gmail.com

Yevgeniy V. Shuvakin

Cand. Sci. (Tech.), Assoc. Prof., Military EducationalResearch Centre of Air Force "Air Force Academy named after professor N.E. Zhukovskiy and Yu. A. Gagarin", Voronezh, Russian Federation, ORCID:

https://orcid.org/0000-0002-1719-9592, E-mail: argentina_85@mail.ru 